\title{
Available Starch: from food process control to lower glycemic response
}

\section{Abstract}

Starch is one of the major sources of carbohydrates (CHO) contributing to about $50-70 \%$ of the daily energy intake in human diet. Processed foods contribute to an important part of daily energy intake, especially in occidental countries. During food processing, starch undergoes dramatic changes when heated in the presence of water or high pressure. The objective of this work was to evaluate the impact of controlling food processes on starch digestibility profile in cereal-based foods and the interest of preserving starch in its native, slowly digestible, form on in vivo metabolic fate.

Four different food processes were evaluated: extrusion, dry bread, soft cake and rotary-moulded biscuit. The resulting differences in gelatinization of each product translated to different SDS levels. Imaging techniques (X-ray diffraction, microtomography and electronic microscopy) were used to investigate the changes in SDS structure obtained through the different food processes. For in vivo evaluation, 12 healthy subjects were recruited and consumed a portion of $50 \mathrm{~g}$ of available $\mathrm{CHOs}$ of each product, in a crossover design. Glycemic and insulin responses were evaluated over 120 minutes in postprandial period.

We showed that rotary molding technology preserved starch in its intact form and provided the highest SDS content $(28 \mathrm{~g} / 100 \mathrm{~g})$ when the three other technologies (dried bread, soft cake and extrusion) led to SDS content below $3 \mathrm{~g} / 100 \mathrm{~g}$. In addition, the lowest SDS contents are explained by a higher level of starch structure disruption which translated in a shift from crystalline structure into amorphous one. These data were linked to in vivo results. The global analysis for GI indicated that a significant difference existed among the foods' mean GI values $(\mathrm{p}<0.0001)$. Rotary product showed the lowest GI values. The soft, the dried bread had medium GI and extruded product had the highest GI with 77. The global analysis for II indicated that a significant difference existed among the foods' mean II values $(\mathrm{p}<0.0001)$, with the same order as for GI values. In addition, a portion of rotary-molded biscuit decreased significantly the glycemic peak by $1 \mathrm{mM}$ compared to the 3 other products.

Decrease of postprandial glycemia is a meaningful target in the prevention of metabolic diseases. This can be achieved through the modification of dietary factors such as starch digestibility.

\section{Conflict of Interest}

All authors are employed by Mondelez Int 